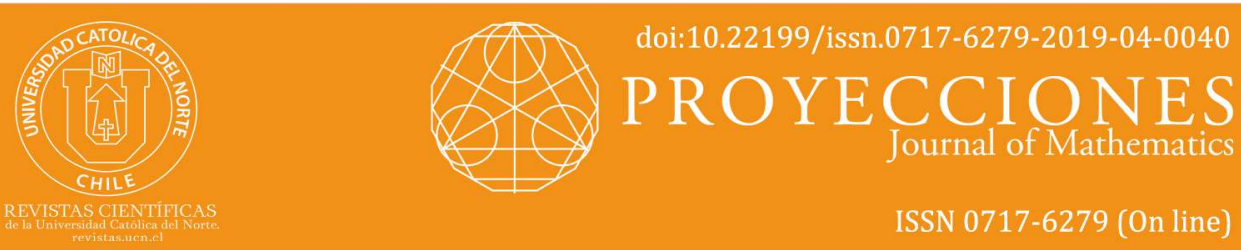

\title{
On flow of electric current in RL circuit using Hilfer type composite fractional derivative
}

\author{
Krunal B. Kachhia* iD orcid.org/0000-0001-8270-7162 \\ J. C. Prajapati** iD orcid.org/0000-0002-5262-3766 \\ K. S. Pandya** (iD orcid.org/0000-0002-0155-000X \\ R. Jadeja ${ }^{* * * *}$ iD orcid.org/0000-0003-3466-203X
}

${ }^{*}$ Charotar University of Science and Technology, Dept. of Mathematics Sciences,

PDPIAS, Changa, GJ, India. $\nabla$ krunalmaths@ hotmail.com

${ }^{* *}$ Sardar Patel University, Dept. of Mathematics, Vallabh Vidyanagar, GJ, India.

jyotindra18@rediffmail.com

${ }^{* * *}$ Charotar University of Science and Technology, Dept. of Electrical Engineering,

Changa, GJ, India. $\nabla$ kartikpandya.ee@charusat.ac.in

${ }^{* * * *}$ Marwadi University, Dept. of Electrical Engineering, Rajkot, GJ, India

\rajendrasinh.jadeja@marwadieducation.edu.in

Received: December 2017 | Accepted: July 2019

\section{Abstract:}

This paper deals with an interdisciplinary research work between Mathematical sciences and Electrical engineering to develop fractional model of Resistance-Inductance circuit $(R L$ circuit). Authors obtained the analytical solution of this fractional model in terms of MittagLeffler function. Graphical interpretation of solution also discussed in this paper.

Keywords: Resistance-Inductance circuit; Fractional differential equation; Mittag-Leffler function; Laplace transforms; Hilfer derivatives.

\section{Cite this article as (IEEE citation style)}

K. Kachhia, J. Prajapati, K. Pandya and R. Jadeja,"On flow of electric current in RL circuit using Hilfer type composite fractional derivative", Proyecciones (Antofagasta, On line), vol. 38, no. 4, pp. 625-636, Oct. 2019, doi: 10.22199/issn.07176279-2019-04-0040. [Accessed dd-mm-yyyy]

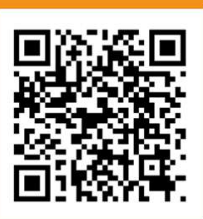

Article copyright: (C) 2019 Krunal Kachhia, Jyotindra Prajapati, Kartik Pandya and Rajendrasinh Jadeja. This is an open access article distributed under the terms of the Creative Commons Licence, which permits unrestricted use and distribution provided the original author and source are credited.

(cc) BY 


\section{Introduction}

Fractional Calculus is one of the generalizations of classical calculus and it has been used successfully in various fields of Science and Technology. Many applications of fractional calculus can be found in other diverse fields, etc. (See [2], [6], [10], [11], [15], [20],[12]). Many works concerning to the electrical components modeling and related to the behavior of the circuits and systems with memristors, meminductors, or memcapacitors have been reported in [25], [9]

Recently, it has been suggested a fractional differential equation that combine the simple harmonic oscillations of an LC circuit with the discharging of an $\mathrm{RC}$ circuit. The behavior of this new hybrid circuit without sources has been analyzed by Rousan et. al [4]. In the work of Obeidat et al. [3] the simple current source-wire circuit has been studied using fractional calculus approach for direct and alternating current source. Guia et al. [18] have done the analysis on the time and frequency domain for the RC electric circuit of fractional order. Shah et al. [19] got analytic solution for the RL electric circuit model in fractional order. Aguilar et al. [14] describes RL and RC circuits using fractional derivative with two fractional orders in LiouvilleCaputo sense.

In this paper, we obtain analytical solutions of the electrical circuits described by Hilfer type composite fractional derivative operators. We use the Laplace transform of getting the analytical solutions of the RL electrical circuits described bycomposite fractional derivative operators.

The simplest electric circuit is a series circuit in which we have a source of electric energy (electromotive force) such as a AC generator or battery, and a resistor, which consume the energy. If we close the switch, a current will flow through the resistor and this will cause a voltage drop, that is, the electric potential at the two ends of the resistor will be different, this potential difference or voltage drop can be measured by a voltmeter.

Current flow in a circuit composed of a battery and conductors can be expressed as ( Toro [28])

$$
I=\frac{A}{\rho} \frac{d v}{d t}
$$

where the derivative term denotes the electric field gradient, which is called the electroscopic force by way of representing the volume density at a point in the conductor. If a conductor of uniform cross-sectional area is used, then (1.1) becomes

$$
I=\frac{A}{\rho} \frac{V}{L}=\frac{V}{R}
$$


where $V$ is the potential difference in volts appearing across the conductor of length $l, A$ is the cross-sectional area, $\rho$ is a property of the material called the resistivity and $R$ is the resistance of the conductor in ohms. Equation (1.2) is a mathematical description of Ohms law. It states that the strength of the current in a wire is proportional to the potential difference between its ends. Ohms law may be alternatively expressed as

$$
V=I R
$$

The form of the equation reveals that for any given potential difference, the amount of current produced is inversely proportional to the resistance, which in turn is dependent upon the composition of the wire.

The voltage drop $V_{R}$ across a resistor is proportional to the instantaneous current $I$, say

$$
V_{R}=I R
$$

where, the constant of proportionality $R$ is called resistance of the resistor. The current $I$ is measured in amperes, the resistance $\mathrm{R}$ in ohms, and the voltage $V_{R}$ in volts. The voltage drop $V_{L}$ across an inductor is proportional to the instantaneous time rate of change of the current $I$, say

$$
V_{L}=L\left(\frac{d I}{d t}\right)
$$

where, the constant of proportionality $L$ is called the inductance of the inductor and is measured in henrys, time $t$ is measured in seconds. The Kirchhoff's voltage Law can be stated as: The algebraic sum of all the instantaneous voltage drops around any closed loop is zero, or the voltage impressed on a closed loop is equal to the sum of the voltage drops in the rest of the loop.

$$
V_{L}+V_{R}=0
$$

The governing equation of current flow using (1.4), (1.5) and Kirchhoff's voltage law as

$$
L \frac{d I}{d t}+R I=E(t)
$$

where $I$ is the current and $L$ is the inductance.

The Heaviside unit step function $u(t-a)$ is defined as (Kreyzing [8])

$$
u(t-a)=\left\{\begin{array}{l}
0, \text { for } t \leq a \\
1, \text { for } t>a
\end{array}\right.
$$


628 Krunal B. Kachhia, J. C. Prajapati, K. S. Pandya and R. Jadeja

\section{Mathematics Prerequisites}

The right-sided Riemann-Liouville fractional integral of order $\alpha$ (Kilbas et. al [1]) defined as

$$
R L{ }_{a} I_{t}^{\alpha}(f(t))=\frac{1}{\Gamma(\alpha)} \int_{a}^{t}(t-\tau)^{\alpha-1} f(\tau) d \tau,(t>a)
$$

where $R(\alpha)>0$.

The right-sided Riemann-Liouville fractional derivative of order $\alpha$ (Kilbas et. al [1]) defined as

$(2.2)^{R L}{ }_{a} D_{t}^{\alpha}(f(t))=\left(\frac{d}{d t}\right)^{n}\left({ }^{R L}{ }_{a} I_{t}{ }^{n-\alpha} f(t)\right)(R(\alpha)>0, n=[R(\alpha)]+1)$,

where $[\alpha]$ represents the integer part of the number $\alpha$.

The following fractional derivative of order $\alpha>0$ (Caputo [17]) defined as

$$
C_{a} D_{t}{ }^{\alpha}(f(t))=\left\{\begin{array}{l}
\frac{1}{\Gamma(m-\alpha)} \int_{a}^{t} \frac{f^{m}(\tau)}{(t-\tau)^{\alpha+1-m}} d \tau, \text { for } m-1<\alpha<m \\
\frac{d^{m} f(t)}{d t^{m}}, \text { for } \alpha=m
\end{array}\right.
$$

$m \in N$ and $\frac{d^{m} f(t)}{d t^{m}}$ is the $m$-th derivative of the function $f(t)$ with respect to $t$.

A generalization of the Riemann-Liouville fractional derivative operator (2.2) and Caputo fractional derivative operator (2.3) is given by Hilfer [20], by introducing a fractional derivative operator of two parameters of order $0<\mu<1$ and type $0 \leq \nu \leq 1$ in the form

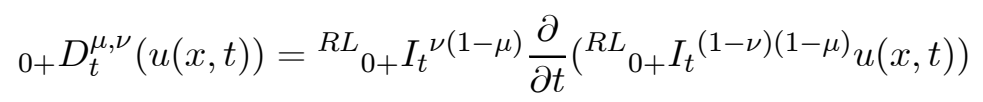

Observed that for $\nu=0,(2.4)$ reduces to the classical Riemann-Liouville fractional derivative operator (2.2). On the other hand, for $\nu=1$ it gives the Caputo fractional derivative operator defined by (2.3).

The Laplace transform for this operator (Hilfer [20]) defined as

$$
L\left\{0+D_{t}^{\mu, \nu}(u(x, t)) ; s\right\}=s^{\mu} L\{u(x, t)\}-s^{\nu(\mu-1) R L_{0+}} I_{t}^{(1-\nu)(1-\mu)} u(x, 0+),(0<\mu<1)
$$

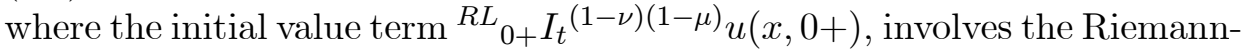
Liouville fractional integral operator of order $(1-\nu)(1-\mu)$ evaluated in the limit as $t \rightarrow 0+$. 
The composite fractional derivative appeared in the theoretical modeling of broadband dielectric relaxation spectroscopy for glasses (Hilfer [21]). Kachhia and Prajapati [15] used composite fractional derivative to study heat transfer through diathermanous materials. Saxena et al. ([23],[22]) obtained analytical solution of some non-linear equations with composite fractional derivatives. Tomovski et al. [29] studied fractional diffusion equation with composite fractional derivatives. Recently Dubbeldam et al. [13] studied fractional Schrödinger equation with composite fractional derivatives. Sandev et al. [26] obtained the solution of a fractional diffusion equation with a Hilfer-generalized Riemann-Liouville time fractional derivative and investigated the solution of generalized distributed order diffusion equations with composite time fractional derivative in [27].

The two parameter Mittag-Leffler function (Wiman [7]) defined as

$$
E_{\alpha, \beta}(z)=\sum_{n=0}^{\infty} \frac{z^{n}}{\Gamma(\alpha n+\beta)}, \alpha, \beta \in C, \operatorname{Re}(\alpha)>0
$$

The Mittag-Leffler function is a direct generalization of the exponential function to which it reduces for $\alpha=\beta=1$. For $0<\alpha<1$ it interpolates between the pure exponential and a hypergeometric function $\frac{1}{1-z}([5])$. The importance of Mittag-Leffler function is realized during the last two decades due to its direct involvement in the problems of physics, biology, engineering and applied sciences. It is naturally occurs as the solution of fractional order differential equations or fractional order integral equations([16],[11]).

\section{Formulation of Fractional Differential Equation Models for Flow of Electricity in RL circuit}

In this section, we developed the resistance-inductance circuit model (1.7) in the form of fractional differential equation as

$$
{ }_{0+} D_{t}^{\mu, \nu}(I(t))+\frac{R}{L} I(t)=\frac{E(t)}{L}
$$

where ${ }_{0+} D_{t}^{\mu, \nu}(I(t))$ is Hilfer type composite fractional derivative of $I(t)$ defined by (2.4). If we take $\mu \rightarrow 1$ and $\nu=1$, then (3.1) reduces to classical case (1.7) discussed in [19].

\section{Solution of Problem}

In this section, authors obtained the solution of various cases of the Resistanceinductance circuit model (3.1). 
630 Krunal B. Kachhia, J. C. Prajapati, K. S. Pandya and R. Jadeja

4.1. Zero electromotive force i.e $E(t)=0$

$$
{ }_{0+} D_{t}^{\mu, \nu}(I(t))+\frac{R}{L} I(t)=0
$$

the initial condition is

$$
R L_{0+} I_{t}^{(1-\nu)(1-\mu)} I(0+)=I_{0},
$$

where $I_{0}$ is constant. Applying Laplace transform on (4.2) and use of (2.5), we get

$$
s^{\mu} \bar{I}(s)-s^{\nu(\mu-1) R L_{0+}} I_{t}^{(1-\nu)(1-\mu)} I(0+)+\frac{R}{L} \bar{I}(s)=0 .
$$

use of (4.2), gives

$$
\bar{I}(s)=I_{0} \frac{s^{\nu(\mu-1)}}{s^{\mu}+\frac{R}{L}}
$$

Inverse Laplace transform of (4.4) is given by (Purohit [24])

$$
I(t)=I_{0} t^{\mu+\nu(1-\mu)-1} E_{\mu, \mu+\nu(1-\mu)}\left(-\frac{R}{L} t^{\mu}\right)
$$

4.2. Constant electromotive force i.e. $E(t)=E_{0}$

In this case (3.1) reduces to

$$
{ }_{0+} D_{t}^{\mu, \nu}(I(t))+\frac{R}{L} I(t)=\frac{E_{0}}{L}
$$

and initial condition is

$$
R L_{0+} I_{t}^{(1-\nu)(1-\mu)} I(0+)=I_{0} .
$$

Applying Laplace transform on (4.6) and use of (2.5), we yield

$$
s^{\mu} \bar{I}(s)-s^{\nu(\mu-1) R L}{ }_{0+} I_{t}^{(1-\nu)(1-\mu)} I(0+)+\frac{R}{L} \bar{I}(s)=\frac{E_{0}}{L} \frac{1}{s}
$$

use of (4.7), gives

$$
\bar{I}(s)=\frac{E_{0}}{L} \frac{1}{s\left(s^{\mu}+\frac{R}{L}\right)}+I_{0} \frac{s^{\nu(\mu-1)}}{s^{\mu}+\frac{R}{L}} .
$$

Inverse Laplace transform of (4.9) gives

$$
\text { (4.10) } I(t)=\frac{E_{0}}{R}\left(1-E_{\mu, 1}\left(-\frac{R}{L} t^{\mu}\right)\right)+I_{0} t^{\mu+\nu(1-\mu)-1} E_{\mu, \mu+\nu(1-\mu)}\left(-\frac{R}{L} t^{\mu}\right)
$$




\subsection{Constant electromotive force in terms of unit step function applied i.e. $E(t)=u(t)$}

In this case (3.1) becomes

$$
{ }_{0+} D_{t}^{\mu, \nu}(I(t))+\frac{R}{L} I(t)=\frac{u(t)}{L}
$$

and initial condition is

$$
{ }^{R L}{ }_{0+} I_{t}^{(1-\nu)(1-\mu)} I(0+)=I_{0} .
$$

Applying Laplace transform on (4.11) and use of (2.5), leads to

$$
s^{\mu} \bar{I}(s)-s^{\nu(\mu-1) R L_{0+}} I_{t}^{(1-\nu)(1-\mu)} I(0+)+\frac{R}{L} \bar{I}(s)=\frac{1}{L} \frac{1}{s}
$$

use of (4.12), gives

$$
\bar{I}(s)=\frac{1}{L} \frac{1}{s\left(s^{\mu}+\frac{R}{L}\right)}+I_{0} \frac{s^{\nu(\mu-1)}}{s^{\mu}+\frac{R}{L}} .
$$

Inverse Laplace transform of (4.9) yields

$$
I(t)=\frac{1}{R}\left(1-E_{\mu, 1}\left(-\frac{R}{L} t^{\mu}\right)\right)+I_{0} t^{\mu+\nu(1-\mu)-1} E_{\mu, \mu+\nu(1-\mu)}\left(-\frac{R}{L} t^{\mu}\right)
$$

4.4. Periodic electromotive force i.e. $E(t)=E_{0} \sin \omega t$

In this case (3.1) can be written in the form

$$
{ }_{0+} D_{t}^{\mu, \nu}(I(t))+\frac{R}{L} I(t)=\frac{E_{0} \sin \omega t}{L}
$$

and initial condition is

$$
R L_{0+} I_{t}^{(1-\nu)(1-\mu)} I(0+)=I_{0} .
$$

Applying Laplace transform on (4.11) and use of (2.5), we get

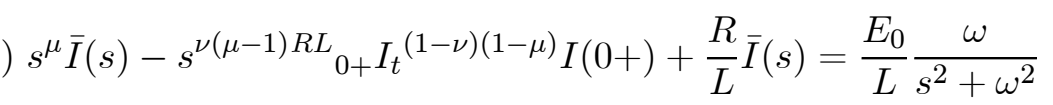

Use of (4.17), gives

$$
\bar{I}(s)=\frac{E_{0}}{L} \frac{1}{s^{\mu}+\frac{R}{L}} \frac{\omega}{s^{2}+\omega^{2}}+I_{0} \frac{s^{\nu(\mu-1)}}{s^{\mu}+\frac{R}{L}} .
$$


632 Krunal B. Kachhia, J. C. Prajapati, K. S. Pandya and R. Jadeja

The inverse Laplace transform of (4.19), we arrived at

$I(t)=\frac{E_{0}}{L} \int_{0}^{t} \tau^{\mu-1} \sin (\omega(t-\tau)) E_{\mu, \mu}\left(-\frac{R}{L} \tau^{\mu}\right) d \tau+I_{0} t^{\mu+\nu(1-\mu)-1} E_{\mu, \mu+\nu(1-\mu)}\left(-\frac{R}{L} \tau^{\mu}\right)$

\section{Results and Discussions}

We get following graphs of time verses current for different values of $\nu$.
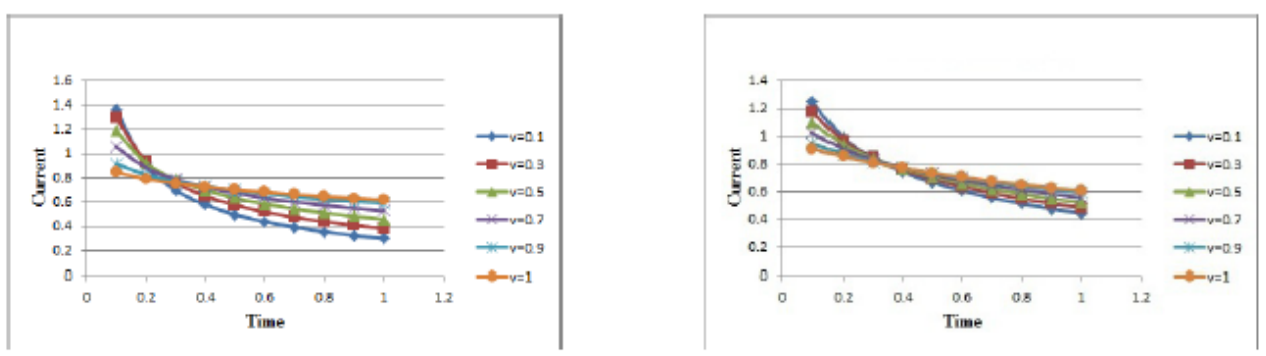

Figure 1: For $\frac{R}{L}=0.5, I_{0}=1, \mu=0.5$ and Figure 2: For $\frac{R}{L}=0.5, I_{0}=1, \mu=0.75$ and $E(t)=0$ that is no electromotive force is ap- $E(t)=0$ that is no electromotive force is applied plied

We get following graphs of time verses current for different values of $\nu$.

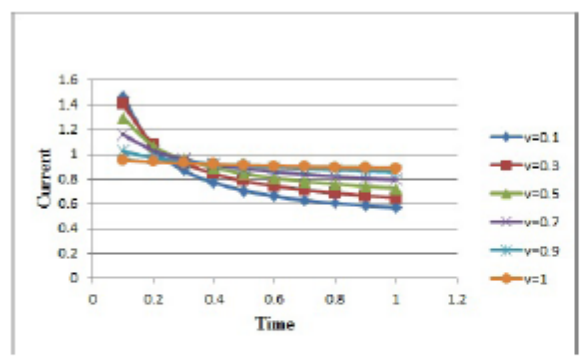

Figure 3: For $\frac{R}{L}=0.5, I_{0}=1, \mu=0.5$ and $E(t)=0$ that is $\operatorname{constant}\left(E_{0}\right)$ electromotive force is applied

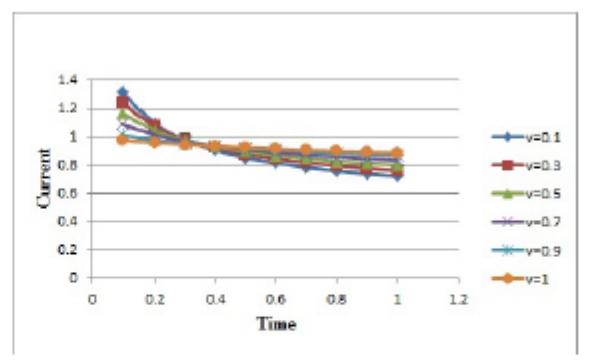

Figure 4: For $\frac{R}{L}=0.5, I_{0}=1, \mu=0.75$ and $E(t)=0$ that is constant $\left(E_{0}\right)$ electromotive force is applied 
Above graphs, observed that the behavior of current by using fractional calculus approach for different values of $\mu$ and $\nu$. When $\nu=0.1$, the current decreases sharply and its moves towards stability as time increases. This exhibit the behavior of current for different values of $\mu, \nu$ with respect to time $(t)$ before the attainment of its natural behavior.

The results showed in figures briefly show that when $\mu=\nu=1$, the system displays the Markovian nature. However, for values of $\mu<1$, the equations describe non-conservative behaviors (non-local in time); in these cases, the voltage or the current changes because of the order derivative $\mu$ and $\nu$ (causing irreversible dissipative effects such as ohmic friction), the different $\mu$ and $\nu$ values exhibit fractional time components (such components change the time constant of the system). The time fractional differentiation represents a non-local effect of dissipation of energy (internal friction) represented by the fractional order $\mu$ and $\nu$.

\section{Conclusion}

This article provides an insight in an application of fractional calculus in a circuit theory from the aspect of their analysis in Laplace domain. We have obtained the analytic solution of the fractional differential equation associated with a RL electrical circuit in time domain using Hilfer type composite fractional derivative in terms of Mittag-Leffler type function which can be implemented for computational study of behaviour of current. Analytical solutions were obtained Laplace transforms. With the approach presented here, it will be possible to have a better study of the transist effects in the electrical system. We conclude that the methodology applied in this paper can be extended for other electrical systems.

\section{Acknowledgement}

Authors are grateful to the referees/reviewers for their valuable comments and suggestions for the betterment of paper.

\section{References}

[1] A. Kilbas, H. Srivastava, and J. Trujillo, Eds., Theory and applications of fractional differential equations. Amsterdam: Elsevier, 2006. [On line]. Available: http://bit.ly/2lLbn3L 
[2] A. Carpinteri and F. Mainardi, Eds., Fractals and fractional calculus in continuum mechanics. Springer: Vienna, 1997, doi:10.1007/978-3-70912664-6.

[3] A. Obeidat, M. Gharaibeh, M. Al-Ali, and A. Rousan, "Evolution of a current in a resistor," Fractional calculus and applied analysis, vol. 14, no. 2, pp. 247-259, Jan. 2011, doi: 10.2478/s13540-011-0015-7.

[4] A. Rousan, N. Ayoub, A. Alzoubi, H. Khateeb, M. Al-Qadi, M. Hasan and B. Albiss, "A fractional LC-RC circuit", Fractional calculus and applied analysis, vol. 9, no. 1, pp. 33-41, 2006. [On line]. Available: http://bit.ly/2mugndn

[5] A. Shukla and J. Prajapati, "On a generalization of Mittag-Leffler function and its properties", Journal of mathematical analysis and applications, vol. 336, no. 3, pp. 797-811, Dec. 2007, doi: 10.1016/j.jmaa.2007.03.018.

[6] I. Jeses and J. Machado, "Fractional control of heat diffusion systems", Nonlinear dynamics, vol. 54, no. 3, pp. 263-282, Dec. 2008, doi: 10.1007/s11071-007-9322-2.

[7] A. Wiman, "Über den fundamentalsatz in der teorie der funktionen $\mathrm{Ea}(\mathrm{x})$ ", Acta Mathematica, vol. 29,no. 1, pp. 191-201, 1905, doi: 10.1007/BF02403202.

[8] E. Kreyszig, Advanced engineering mathematics, 8th ed. New York (NY): John Wiley \& Sons, Inc, 2007.

[9] G. Tsirimokou and C. Psychalinos, "Ultra-low voltage fractional-order circuits using current mirrors", International journal of circuit theory and applications, vol. 44, no. 1, pp. 109-126, Feb. 2015, doi: 10.1002/cta.2066.

[10] I. Podulbuny, Fractional differential equations, New York (NY): Academic Press, 1999.

[11] J. Prajapati and K. Kachhia, "Fractional modeling of temperature distribution and heat flux in the semi infinite solid", Journal of fractional calculus and applications, vol. 5, no. 2, pp. 38-43, Jul. 2014. [On line]. Available: http://bit.ly/2miHCb2

[12] J. Prajapati, K. Kachhia, and S. Kosta, "Fractional calculus approach to study temperature distribution within a spinning satellite," Alexandria engineering journal, vol. 55, no. 3, pp. 2345-2350, Sep. 2016, doi: 10.1016/j.aej.2016.05.004.

[13] J. Dubbeldam, Z. Tomovski and T. Sandev, "Space-Time Fractional Schrödinger equation with composite time fractional derivative", Fractional calculus and applied analysis, vol. 18, no. 5, pp. 1179-1200, Oct. 2015, doi: 10.1515/fca-2015-0068.

[14] J. Gómez-Aguilar, R. Escobar-Jiménez, V. Olivares-Peregrino, M. TanecoHernández, and G. Guerrero-Ramírez, "Electrical circuits RC and RL involving fractional operators with bi-order," Advances in mechanical engineering, vol. 9, no. 6, pp. 1-10, Jun. 2017, doi: 10.1177/1687814017707132. 
[15] K. Kachhia and J. Prajapati, "Solution of fractional partial differential equation aries in study of heat transfer through diathermanous materials", Journal of interdisciplinary mathematics, vol. 18, no. 1-2, pp. 125-132, Mar. 2015, doi: 10.1080/09720502.2014.996017.

[16] K. Kachhia and J. Prajapati, "On generalized fractional kinetic equations involving generalized Lommel-Wright functions", Alexandria engineering journal, vol. 55, no. 3, pp. 2953-2957, Sep. 2016, doi: 10.1016/j.aej.2016.04.038.

[17] M. Caputo, Elasticita e dissipazione, Bologna: Zanichelli, 1969.

[18] M. Guía, F. Gómez, and J. Rosales, "Analysis on the time and frequency domain for the RC electric circuit of fractional order", Open physics, vol. 11, no. 10, Oct. 2013, doi: 10.2478/s11534-013-0236-y.

[19] P. Shah, A. Patel, I. Salehbhai, and A. Shukla, "Analytic solution for the RL electric circuit model in fractional order", Abstract and applied analysis, vol. 2014, ID 343814, Jun. 2014, doi: 10.1155/2014/343814.

[20] R. Hilfer, Applications of fractional calculus in physics, Singapore: World scientific, 2000.

[21] R. Hilfer, "Experimental evidence for fractional time evolution in glass forming materials," Chemical physics, vol. 284, no. 1-2, pp. 399-408, Nov. 2002, doi: 10.1016/S0301-0104(02)00670-5.

[22] R. Saxena, A. Mathai, and H. Haubold, "Space-time fractional reaction-diffusion equations associated with a generalized Riemann-Liouville fractional derivative", Axioms, vol. 3, no. 3, pp. 320-334, Aug. 2014, doi: 10.3390/axioms3030320.

[23] R. Saxena, Z. Tomovski and T. Sandev, "Fractional Helmholtz and fractional wave equations with Riesz-Feller and generalized Riemann-Liouville fractional derivatives", European journal of pure and applied mathematics, vol. 7, no. 3, pp. 312-334, Aug. 2014. [On line]. Available: http://bit.ly/2nZzigI

[24] S. Purohit, "Solution of fractional partial differential equations of quantum mechanics", Advances in Applied Mathematics and Mechanics, vol. 5, no. 5, pp. 639-651, Oct. 2011, doi: 10.1017/S2070073300002356.

[25] T. Kaczorek and K. Rogowski, "Positive fractional electrical circuits", in Fractional Linear Systems and Electrical Circuits, vol. 13, Cham: Springer, 2015, pp. 49-80, doi: 10.1007/978-3-319-11361-6_2.

[26] T. Sandev, R. Metzler, and Ž. Tomovski, "Fractional diffusion equation with a generalized Riemann-Liouville time fractional derivative",Journal of physics A: mathematical and theoretical, vol. 44, no. 25, p. 255203, May 2011, doi: 10.1088/1751-8113/44/25/255203.

[27] T. Sandev, Z. Tomovski, and B. Crnkovic, "Generalized distributed order diffusion equations with composite time fractional derivative", Computers \& mathematics with applications, vol. 73, no. 6, pp. 1028-1040, Mar. 2017, doi: 10.1016/j.camwa.2016.07.009. 
636 Krunal B. Kachhia, J. C. Prajapati, K. S. Pandya and R. Jadeja

[28]V. Toro, Electrical engineering fundamentals, 2nd ed. Delhi: Prentice-Hall, 2002.

[29]Ž. Tomovski, T. Sandev, R. Metzler, and J. Dubbeldam, “Generalized space-time fractional diffusion equation with composite fractional time derivative", Physica A: Statistical Mechanics and its Applications, vol. 391, no. 8, pp. 2527-2542, Apr. 2012, doi: 10.1016/j.physa.2011.12.035. 